\begin{tabular}{|c|l|}
\hline Title & CD133 Negatively Regulates Tumorigenicity via A KT Pathway in Synovial Sarcoma \\
\hline Author(s) & $\begin{array}{l}\text { Kimura, Taichi; Wang, Lei; Tabu, Kouichi; Nishihara, Hiroshi; Mashita, Yuji; Kikuchi, Naoyuki; Tanino, Mishie; } \\
\text { Hiraga, Hiroaki; Tanaka, Shinya }\end{array}$ \\
\hline Citation & $\begin{array}{l}\text { Cancer Investigation, 30(5), 390-397 } \\
\text { https://doi.org/10.3109/07357907.2012.672607 }\end{array}$ \\
\hline Issue Date & 2012-06 \\
\hline Doc URL & http://hdl.handle.net/2115/52796 \\
\hline Type & article(author version) \\
\hline File Information & Cl30-5_390-397.pdf \\
\hline
\end{tabular}

Instructions for use 


\section{CD133 negatively regulates tumorigenicity via AKT pathway in synovial}

\section{sarcoma}

Taichi Kimura ${ }^{1}$, Lei Wang ${ }^{1}$, Kouichi Tabu ${ }^{2}$, Hiroshi Nishihara ${ }^{3}$, Yuji Mashita ${ }^{1}$, Naoyuki Kikuchi $^{1}$, Mishie Tanino ${ }^{1}$, Hiroaki Hiraga ${ }^{4}$, Shinya Tanaka ${ }^{1,3,5}$

${ }^{1}$ Laboratory of Cancer Research, Department of Pathology, Hokkaido University Graduate School of Medicine, N15, W7, Sapporo, ${ }^{2}$ Department of Stem Cell Regulation, Medical Research Institute, Tokyo Medical and Dental University, 1-5-45 Yushima, Bunkyo-ku, Tokyo, ${ }^{3}$ Department of Translational Pathology, Hokkaido University Graduate School of Medicine, N15, W7, Sapporo, ${ }^{4}$ Division of Orthopedics, National Hospital Organization Hokkaido Cancer Center, Sapporo, Japan

${ }^{5}$ Corresponding author: Shinya Tanaka, M.D., Ph.D.

Laboratory of Cancer Research, Department of Pathology, Hokkaido University Graduate School of Medicine,

N15, W7, Sapporo, 060-8638, Japan

Tel: $\quad+81-11-706-5052$

Fax: $\quad+81-11-706-5902$

e-mail: tanaka@med.hokudai.ac.jp

Running title: Functional analysis of CD133 in synovial sarcoma 


\begin{abstract}
Synovial sarcoma is an aggressive tumor which accounts for almost $10 \%$ of all soft tissue sarcomas. In this study, we found the expression of CD133 in human synovial sarcoma specimens, thus we focused on the function of CD133 in synovial sarcoma. Separation of the CD133-positive and -negative subpopulations in synovial sarcoma cell lines clarified that the CD133-negative subpopulation exhibited enhanced growth and hyperphosphorylation of AKT. Treatment of Akt inhibitor suppressed the cell growth of CD133-negative subpopulation to the levels of CD133-positive cells. These results suggest that CD133 has negative effect on the growth of cells through AKT-dependent signalling pathway.
\end{abstract}

Key Words: Synovial sarcoma, cancer stem cell, CD133 


\section{Introduction}

Synovial sarcoma accounts for almost $10 \%$ of all soft tissue sarcomas and typically arises in the para-articular regions in adolescents and young adults. Synovial sarcoma has been shown to be caused by chimeric oncogene product SS18-SSXresulting from chromosomal translocation $\mathrm{t}(18 ; \mathrm{X})$. Synovial sarcoma is a misnomer because its origin is not identical to the synovium [1]. Currently, synovial sarcoma is thought to arise from undifferentiated mesenchymal cells. Prognosis of synovial sarcoma is poor as the 5- and 10year survival rates are $36 \%$ and $20 \%$, respectively [2].

The precise mechanism of transforming activity of SS18-SSX is not clearly understood. As SS18 bound to several proteins as chromatin remodeling factor hBRM, histone acetyl transferase p300, or a component of histone deacetylase complex mSin3A, SS18-SSX may disturb epigenetical regulation and this may be contributed to the transforming potential of SS18-SSX $[1,3,4]$. For the malignant potential of invasion or metastasis, tyrosine kinases as Src or Met and its downstream adaptor molecule Crk may be involved [5-7].

Stem-like cells in malignant tumors have been shown to be involved in tumor formation, maintenance, chemoresistance, and radiation resistance [8]. In contrast to the extensive research for cancer stem cells in epithelial malignancies as carcinoma such as colon cancer, breast cancer, prostate cancer, etc., only a few studies have been reported for the fields of mesenchymal malignancy as sarcoma [9-11]. In particular, there are fewer reports 
investigating whether stem-like cells exist in synovial sarcoma.

Among various stem cell markers, CD133 has been shown to play an important role in various cancers including glioma, prostate cancer, pancreatic cancer, colon cancer, and hepatocellular carcinoma [12-16]. In addition, epigenetical regulation of CD133 was reported to be involved in glioma $[17,18]$. As SS18-SSX may be involved in epigenetical regulations, thus, we investigated the role for CD133, in synovial sarcoma. 


\section{Materials and Methods}

\section{Cell culture and reagents}

The human colon carcinoma cell line Caco-2 and synovial sarcoma cell lines FUJI, HS-SY II, and SYO-1 were cultured in DMEM containing 10\% fetal bovine serum (FBS) supplemented with $2 \mathrm{mM} \mathrm{L-glutamine,} 100 \mathrm{U} / \mathrm{ml}$ penicillin G, and $100 \mu \mathrm{g} / \mathrm{ml}$ streptomycin, at $37^{\circ} \mathrm{C}\left(5 \% \mathrm{CO}_{2}\right)$. Cells were treated with the AKT inhibitor VIII (Calbiochem, San Diego, CA) at $1.0 \mu \mathrm{M}$ for 5 days with renewal of media every 24 hours.

\section{Histopathological and immunohistochemical analysis}

Five specimens of formalin-fixed, paraffin-embedded human monophasic synovial sarcoma diagnosed in Hokkaido Cancer Center were used under the certification of the ethical committee. The human tumor specimens and the mice xenografts of the cell line in nude mice were sectioned and stained with hematoxylin and eosin (H\&E) using standard protocol. Immunohistochemistry was performed using anti-CD133 (Abcam, Cambridge, UK) antibody.

\section{Semi-quantitative RT-PCR}

Total RNA was isolated from synovial sarcoma specimens and all cell lines by using RNeasy mini kit (Qiagen, CA, USA) according to the manufacturer's instructions. Reverse transcription was carried out with Superscript II RT (Invitrogen, CA, USA). One hundred ng of the resulting first-strand cDNA was used as a template and amplified by PCR using GoTaq Green Master Mix (Promega, WI, USA). Sequences of the oligonucleotide primer sets used 
for RT-PCR are as follows: CD133: Forward (5'- TGGCAACAGCGATCAAGGAGAC -3'), reverse (5'- TCGGGGTGGCATGCCTGTCATA -3'); SS18-SSX1: Forward (5'CAACAGCAAGATGCATACCA -3'), reverse (5'- AGATCTCTTATTAATCTTCTCAGAAA -3'); SS18-SSX2: Forward (5' - CAACAGCAAGATGCATACCA -3'), reverse (5'TTTTGGGTCCAGATCTCTCGTG -3'); CD73: Forward (5'ACAGCTATGTGTCCCCGAGC -3'), reverse (5'- GATGCTAGTGGCCCCTTTGC -3’); CD90: Forward (5'- ACCTGGCCATCAGCATCGCT -3'), reverse (5'TTCCTGTCTCCTCCATGGGC -3’); CD105: Forward (5'-

CAGCCCCACAAGTCTTGCAG -3'), reverse (5'- TGCAGAAGGACAGTGACCCC -3'); SS18: Forward (5'- CAACAGCAAGATGCATACCA -3'), reverse (5'TCACTGCTGGTAATTTCCAT -3'); and GAPDH: Forward (5'CTCATGACCACAGTCCATGC -3'), reverse (5'- TTACTCCTTGGAGGCCATGT -3').

\section{SDS-PAGE and immunoblotting}

SDS-PAGE and immunoblotting were performed as described previously [19].

Antibodies (Abs) were obtained from the following sources: anti-CD133 Ab (Miltenyi Biotec,

CA, USA), anti- $\alpha$ Tubulin Ab (SIGMA, MI, USA), anti-p300, anti-CDK4, and anti-CyclinD1 Abs (Santa Cruz Biotechnology, CA, USA), anti-BRG1 and anti-hBRM Abs (kindly gifted from Yasunori Machida in Nagoya Univ., Japan), anti- SS18 Ab (generated in our lab), antiACTIN Ab (Chemicon International, CA, USA), anti-phospho-AKT, anti-AKT, anti-phospho- 
ERK, anti-phospho-JNK, anti-phospho-cJUN, and anti-phospho-p38 Abs (Cell Signaling Technology, MA, USA), anti-CDK1 and anti-CDK2 Abs (BD Biosciences, CA, USA).

\section{Magnetic and fluorescence activated cell sorting}

For enrichment of CD133-expressing cells, FUJI and SYO-1 cells were subjected to immunomagnetic separation by using a magnetic activated cell sorting (MACS) CD133 Cell Isolation Kit (Miltenyi Biotec), according to the manufacturer's protocol. To check the expression of CD133, cells were stained with monoclonal antibody for human CD133 (CD133/2, Miltenyi Biotec) or isotype control antibody IgG2b (Miltenyi Biotec), followed by incubation with Alexa Fluor 488 Goat anti-mouse antibody (Invitrogen, CA, USA). After washing, the labeled cells were analyzed by a BD FACSCalibur flow cytometer (BD Biosciences, CA, USA).

\section{Xenograft propagation}

Subcutaneously, $5 \times 10^{6}$ cells were injected with matrigel into three of the 3 -week-old female nude mice, BALB/cA Jcl-nu (nu/nu) (CLEA Japan Inc., Japan). The mice were euthanized 22 days after injection, and tumors were removed and weighed. Histopathological examinations were performed. 


\section{Results}

To evaluate the presence of CD133-positive cells in human synovial sarcoma, immunohistochemical analysis of surgical specimens which were diagnosed as monophasic synovial sarcoma was performed and CD133-positive cells were detected with lower frequency in all examined specimens (Figure 1a). CD133 mRNA was also detectable in human synovial sarcoma at various levels (Figure 1b). To assess the function of CD133 in vitro and in vivo, we used human synovial sarcoma cell lines such as FUJI and SYO-1, in which expressions of both mRNA and protein of CD133 were confirmed (Figure 1c and 1d). Furthermore, FACS analysis using anti-CD133 antibody detected CD133 expression in both cell lines (Figure 1e). These results suggest that synovial sarcoma consists of a heterogeneous population in terms of CD133 expression.

To analyze the function of CD133 in synovial sarcoma cell lines, we enriched CD133-positive and -negative subpopulations of cells of FUJI and SYO-1 by using immuneaffinity beads as MACS system. CD133-positive subpopulation of SYO-1 cells could be enriched about 13 times to CD133-negative subpopulation (76.5\% vs. 5.94\%), and in FUJI cells, the enrichment ratio was 11 folds (82\% vs. 7.2\%), (Figure 2a).

For the initial step to evaluate biological differences of CD133-positivity, we examined the expression levels for SS18 interacting proteins such as p300, BRG1, and hBRM in CD133-positive and negative cells, but we could not detect any significant change of these 
protein levels (Figure 2b). To examine whether CD133 plays a role for stemness in synovial sarcoma, we confirmed the expression of several mesenchymal stem cell (MSC) markers, which are the candidates for the origin of various sarcomas $[9,11,20,21]$. However, no significant difference of the levels of MSC markers was observed among CD133positive/negative subpopulations of synovial sarcoma (Figure 2c). The levels of SS18-SSX1 were not correlated with the amount of CD133 (Figure 2c). These data suggest that MSC marker may not be associated with CD133-positive subpopulation in synovial sarcoma cell lines.

To analyze the effect of CD133 on cell proliferation, we analyzed the growth curve of each subpopulation. In both FUJI and SYO-1 cells, growth rate of the CD133-negative population was significantly higher than that of the CD133-positive subpopulation (Figure 3a and $3 \mathrm{~b}$ ). Correlating to these results, the in vivo tumorigenicity of the CD133-positive cells was lower than that of the CD133-negative subpopulations in FUJI and SYO cells (Figure 3c and 3d). Histopathologically, the tumors of all xenografts exhibited the characteristic features of human synovial sarcoma (Figure 3c).

To analyze the downstream signaling pathways of CD133 regulating cell growth and tumorigenicity, we examined the levels of several kinases associated with cell proliferation or survival including MAP kinases, cyclin, cyclin dependent kinases, and AKT. The increased phosphorylation levels of AKT were found in the CD133-negative subpopulation of both 
SYO-1 (Figure 4a) and FUJI cells (data not shown). To assess whether the hyperphosphorylation of AKT positively regulates cell growth of the CD133-negative subpopulation, we analyzed the effect of AKT inhibitor on synovial sarcoma cell lines. The growth rate of the CD133-negative subpopulation treated with AKT inhibitor was decreased to the same level as that of untreated CD133-positive subpopulation (Figure 4c). The inhibitory effect of AKT inhibitor was subtle in case of CD133-positive cells (Figure 4c). These results indicate that AKT pathway may regulate the growth facilitation of the CD133negative subpopulation. 


\section{Discussion}

To elucidate the molecular function of CD133 in synovial sarcoma, we analyzed CD133positive and -negative subpopulations of synovial sarcoma cell lines and found that CD133negative subpopulation exhibited high cell proliferation and tumorigenicity associated with AKT hyperphosphorylation. To our knowledge, this is the first report for the analysis of CD133 in synovial sarcoma and the correlation of CD133 to AKT phosphorylation. The observation of phosphorylation of AKT in human synovial sarcoma tissue specimens ensures our data obtained by cell line study. CD133 is serpentine receptor but currently the ligands for CD133 or CD133-dependent signaling pathway is not clearly understood. There is one report that CD133 may enhance tyrosine kinase Src [22]. As we have also found that the levels of Src is altered by CD133 in some condition (data not shown), Src-family tyrosine kinases may be associated with CD133.

In this study, we examined the relationship between CD133 and mesenchymal stem cell (MSC) markers, however, clear correlation is not obtained. MSC is thought to be one of the potential candidates for the origin of various sarcomas $[10,20,21]$. In fact, a few studies have been reported for the sarcoma stem cells in which the stem-like cells of Ewing sarcoma express CD133 and possess MSC features [11,20,21]. Our results suggest that the CD133positive subpopulation in SS cell lines is not significantly associated with features of MSC, and this suggest that origin of synovial sarcoma is different from that of Ewing sarcoma. Recent report indicating that neural crest cell, which can differentiate both ectoderm and 
mesoderm, is the potential origin of synovial sarcoma [23].

The CD133-negative subpopulation has a higher proliferation rate and tumorigenicity in synovial sarcoma cell lines. To date, the relation of cancer stemness and proliferation activity is controversial and it also remains controversial whether CD133 is a bona fide marker for cancer stem cells [24]. While CD133 is widely used as a marker for cancer stem cells, there is increasing evidence that CD133 negative cancer cells from glioblastoma [25], colon cancer [26], and the Daoy medulloblastoma cell line [27] can form tumors when transplanted in vivo. Moreover, there was no significant correlation of CD133 expression with survival and tumor aggressiveness in glioblastoma [28], esophageal cancer [29], pancreatic cancer [30], and non-small cell lung cancer [31]. Thus, we should carefully consider the generalization of our negative effect of CD133 on tumor growth. There are a few controversial reports showing that AKT hyperphosphorylation is observed in the CD133-positive stem-like cells of malignant glioma and colon cancer [32,33]. As phosphatidylinositol-3'-kinase/AKT signaling has been reported to be essential in synovial sarcoma $[34,35]$, AKT may be one of the therapeutic targets.

Recently, the presence of subpopulations of CD133 expressing cells in synovial sarcoma was reported [36]. In addition, two synovial sarcoma cell lines were reported to exhibit generate sarcospheres [37]. Thus the role for CD133 in synovial sarcoma is one of the hot topics. As our study suggest that CD133 may not be a stem cell marker for synovial 
sarcoma, the determination of the stem cell marker needs future extensive studies for synovial sarcoma. 


\section{Acknowledgements}

We thank Ms. Miho Nodagashira and Ms. Eiko Aoyanagi for the excellent technical assistance of immunohistochemistry. This work was supported in part by Grant-in-Aid for

Scientific Research on Priority Area 'Cancer' (20013001) from the Ministry of Education,

Culture, Sports, Science and Technology and for Scientific Research (B) (19390386) from the Japan Society for the Promotion of Science. 


\section{References}

1. Nagai, M.; Tanaka, S.; Tsuda, M.; et al. Analysis of transforming activity of human synovial sarcoma-associated chimeric protein SYT-SSX1 bound to chromatin remodeling factor hBRM/hSNF2 alpha. Proc Natl Acad Sci U S A 2001, 98(7), 38433848 .

2. Haldar, M.; Randall, RL.; Capecchi, MR. Synovial sarcoma: from genetics to geneticbased animal modeling. Clin Orthop Relat Res 2008, 466(9), 2156-2167.

3. Eid, J.E.; Kung, A.L.; Scully, R.; Livingston, D.M. p300 interacts with the nuclear proto-oncoprotein SYT as part of the active control of cell adhesion. Cell 2000, 102(6), 839-848.

4. Ito, T.; Ouchida, M.; Ito, S.; et al. SYT, a partner of SYT-SSX oncoprotein in synovial sarcomas, interacts with $\mathrm{mSin} 3 \mathrm{~A}$, a component of histone deacetylase complex. Lab Invest 2004, 84(11), 1484-1490.

5. Tsuda, M.; Watanabe, T.; Seki, T.; et al. Induction of p21(WAF1/CIP1) by human synovial sarcoma-associated chimeric oncoprotein SYT-SSX1. Oncogene 2005, 24(54), 7984-7990.

6. Watanabe, T.; Tsuda, M.; Makino, Y.; et al. Adaptor molecule Crk is required for sustained phosphorylation of Grb2-associated binder 1 and hepatocyte growth factorinduced cell motility of human synovial sarcoma cell lines. Mol Cancer Res 2006, 4(7), 499-510.

7. Watanabe, T.; Tsuda, M.; Makino, Y.; et al. Crk adaptor protein-induced phosphorylation of Gab1 on tyrosine 307 via Src is important for organization of focal adhesions and enhanced cell migration. Cell Res 2009, 19(5), 638-650.

8. Jordan, C.T.; Guzman, M.L.; Noble, M. Cancer stem cells. N Engl J Med 2006, 355(12), 1253-1261.

9. Adhikari, A.S.; Agarwal, N.; Wood, B.M.; et al. CD117 and Stro-1 identify osteosarcoma tumor-initiating cells associated with metastasis and drug resistance. Cancer Res 2010, 70(11), 4602-4612.

10. Rubio, R.; Garcia-Castro, J.; Gutierrez-Aranda, I.; et al. Deficiency in p53 but not retinoblastoma induces the transformation of mesenchymal stem cells in vitro and initiates leiomyosarcoma in vivo. Cancer Res 2010, 70(10), 4185-4194.

11. Suva, M.L.; Riggi, N.; Stehle, J.C.; et al. Identification of cancer stem cells in Ewing's sarcoma. Cancer Res 2009, 69(5), 1776-1781.

12. Singh, S.K.; Hawkins, C.; Clarke, I.D.; et al. Identification of human brain tumour initiating cells. Nature 2004, 432(7015), 396-401.

13. Collins, A.T.; Berry, P.A.; Hyde, C.; Stower, M.J.; Maitland, N.J. Prospective identification of tumorigenic prostate cancer stem cells. Cancer Res 2005, 65(23), 10946-10951. 
14. Olempska, M.; Eisenach, P.A.; Ammerpohl, O.; Ungefroren, H.; Fandrich, F.; Kalthoff, $\mathrm{H}$. Detection of tumor stem cell markers in pancreatic carcinoma cell lines. Hepatobiliary Pancreat Dis Int 2007, 6(1), 92-97.

15. Ricci-Vitiani, L.; Lombardi, D.G.; Pilozzi, E.; et al. Identification and expansion of human colon-cancer-initiating cells. Nature 2007, 445(7123), 111-115.

16. Yin, S.; Li, J.; Hu, C.; et al. CD133 positive hepatocellular carcinoma cells possess high capacity for tumorigenicity. Int J Cancer 2007, 120(7), 1444-1450.

17. Tabu, K.; Kimura, T.; Sasai, K.; et al. Analysis of an alternative human CD133 promoter reveals the implication of Ras/ERK pathway in tumor stem-like hallmarks. Mol Cancer 2010, 9, 39.

18. Tabu, K.; Sasai, K.; Kimura, T.; et al. Promoter hypomethylation regulates CD133 expression in human gliomas. Cell Res 2008, 18(10), 1037-1046.

19. Wang, L.; Tabu, K.; Kimura, T.; et al. Signaling adaptor protein Crk is indispensable for malignant feature of glioblastoma cell line KMG4. Biochem Biophys Res Commun 2007, 362(4), 976-981.

20. Riggi, N.; Suva, M.L.; De, Vito, C.; et al. EWS-FLI-1 modulates miRNA145 and SOX2 expression to initiate mesenchymal stem cell reprogramming toward Ewing sarcoma cancer stem cells. Genes Dev 2010, 24(9), 916-932.

21. Tirode, F.; Laud-Duval, K.; Prieur, A.; Delorme, B.; Charbord, P, Delattre, O. Mesenchymal stem cell features of Ewing tumors. Cancer Cell 2007, 11(5), 421-429.

22. Boivin, D.; Labbe, D.; Fontaine, N.; et al. The stem cell marker CD133 (prominin-1) is phosphorylated on cytoplasmic tyrosine- 828 and tyrosine- 852 by Src and Fyn tyrosine kinases. Biochemistry 2009, 48(18), 3998-4007.

23. Nagayama, S.; Katagiri, T.; Tsunoda, T.; et al. Genome-wide analysis of gene expression in synovial sarcomas using a cDNA microarray. Cancer Res 2002, 62(20), 5859-5866.

24. Nishide, K.; Nakatani, Y.; Kiyonari, H.; Kondo, T. Glioblastoma formation from cell population depleted of Prominin1-expressing cells. PLoS One 2009, 4(8), e6869.

25. Beier, D.; Hau, P.; Proescholdt, M.; et al. CD133(+) and CD133(-) glioblastomaderived cancer stem cells show differential growth characteristics and molecular profiles. Cancer Res 2007, 67(9), 4010-4015.

26. Shmelkov, S.V.; Butler, J.M.; Hooper, A.T.; et al. CD133 expression is not restricted to stem cells, and both $\mathrm{CD133+}$ and CD133- metastatic colon cancer cells initiate tumors. J Clin Invest 2008, 118(6), 2111-2120.

27. Srivastava, V.K.; Nalbantoglu, J. Flow cytometric characterization of the DAOY medulloblastoma cell line for the cancer stem-like phenotype. Cytometry A 2008, 73(10), 940-948.

28. Kim, K.J.; Lee, K.H.; Kim, H.S.; et al. The presence of stem cell marker- 
expressing cells is not prognostically significant in glioblastomas. Neuropathology 2011, 31(5), 494-502.

29. Hang, D.; Dong, H.C.; Ning, T.; Dong, B.; Hou, D.L.; Xu, W.G. Prognostic value of the stem cell markers CD133 and ABCG2 expression in esophageal squamous cell carcinoma. Dis Esophagus 2012, Epub ahead of print.

30. Immervoll, H.; Hoem, D.; Sakariassen, P.O.; Steffensen, O.J.; Molven, A. Expression of the "stem cell marker" CD133 in pancreas and pancreatic ductal adenocarcinomas. BMC Cancer 2008, 8:48.

31. Salnikov, A.V.; Gladkich, J.; Moldenhauer, G.; Volm, M.; Mattern, J.; Herr I. CD133 is indicative for a resistance phenotype but does not represent a prognostic marker for survival of non-small cell lung cancer patients. Int $\mathbf{J}$ Cancer 2010, 126(4), 950-958.

32. Ferrand, A.; Sandrin, M.S.; Shulkes, A.; Baldwin, G.S. Expression of gastrin precursors by CD133-positive colorectal cancer cells is crucial for tumour growth. Biochim Biophys Acta 2009, 1793(3), 477-488.

33. Griffero, F.; Daga, A.; Marubbi, D.; et al. Different response of human glioma tumorinitiating cells to epidermal growth factor receptor kinase inhibitors. J Biol Chem 2009, 284(11), 7138-7148.

34. Bozzi, F.; Ferrari, A.; Negri, T.; et al. Molecular characterization of synovial sarcoma in children and adolescents: evidence of akt activation. Transl Oncol 2008 1(2), 95101.

35. Friedrichs, N.; Trautmann, M.; Endl, E.; et al. Phosphatidylinositol-3'-kinase/AKT signalling is essential in synovial sarcoma. Int J Cancer 2011, 129(7), 1564-75.

36. Terry, J.; Nielsen, T. Expression of CD133 in synovial sarcoma. Appl Immunohistochem Mol Morphol 2010, 18(2), 159-165.

37. Naka, N.; Takenaka, S.; Araki, N.; et al. Synovial sarcoma is a stem cell malignancy. Stem Cells 2010, 28(7), 1119-1131. 


\section{Legend for Figures}

Fig. 1. CD133 expression in human synovial sarcoma (SS) and SS cell lines.

(a) Histological (upper panels) and immunohistochemical (lower panels) analysis of CD133 expression in human SS. (b) Semi quantitative RT-PCR analysis of CD133, SS18-SSX1, and 2 in human SS. (c) Semi quantitative RT-PCR analysis of CD133 in human SS cell lines.

Caco2 is positive control for CD133. DW (template cDNA (-) is negative control. (d) Immunoblot analysis of CD133 in SS cell lines. $\alpha$ Tubulin was loading control. $30 \mu \mathrm{g}$ of proteins were loaded in each lane. (e) Flow cytometry analysis of CD133 in SS cell lines. Black bars and percentage indicate the ratio of CD133-positive subpopulation. Black broken lines indicate isotype matched control. Representative data of three independent experiments are shown.

Fig. 2. Enrichment of CD133-positive subpopulation in SS cell lines and expression of SS18SSX interacting proteins and of mesenchymal stem cell markers in CD133-positive or negative subpopulations. (a) Flow cytometry analysis of CD133-positive or -negative subpopulation in SS cell lines after MACS sorting. Black bars and percentage indicate the ratio of CD133-positive subpopulation. Black broken lines indicate isotype matched control. Representative data of three independent experiments are shown. (b) Immunoblot analysis of 
CD133 and SS18-SSX interacting proteins. ACTIN was loading control. $30 \mu \mathrm{g}$ of proteins were loaded in each lane. (c) Semi quantitative RT-PCR analysis of mesenchymal stem cell markers, SS18, SS18-SSX1, and CD133. Mesenchymal stem cell (MSC) and human fibroblast (TIG3) is positive control for CD133.

Fig. 3. In vitro and in vivo growth facilitation of CD133-negative subpopulation in SS cell lines. Growth curves of CD133-positive and negative subpopulations in FUJI (a) and SYO-1 (b). Data represent the number of viable cells and are means \pm s.d. of values from three independent experiments. ${ }^{*} P<0.05,{ }^{* *} P<0.01,{ }^{* * *} P<0.005$ (Student's $t$-test) versus the corresponding value for CD133-negative subpopulation, respectively. (c) For xenograft propagation, $5 \times 10^{6}$ cells were subcutaneously injected into nude mice (CD133-positive, $n=3$; negative, $n=3$ ). Macroscopic appearance of tumors is also shown (upper panel). Formalin-fixed, paraffin-embedded sections of CD133-positive (lower left) and -negative (lower right) xenografts were subjected to histological analyses. H\&E staining are shown. (d) Statistical analysis of mouse xenografts is shown. Data represent the weight of xenografts and are means \pm s.d. of values from three independent experiments. $P<0.05, P<0.01$, (Student's $t$ test) versus the corresponding value for CD133-negative subpopulation, respectively.

Fig. 4. Growth facilitation of CD133-negative subpopulation in SS cell lines is due to AKT 
hyperphosphorylation. (a) Immunoblot analysis of proliferation-related proteins. ACTIN was loading control. $30 \mu \mathrm{g}$ of proteins were loaded in each lane. (b) Immunoblot analysis of phospho-AKT and total-AKT protein derived from SYO-1 treated by AKT inhibitor. ACTIN was loading control. $30 \mu \mathrm{g}$ of proteins were loaded in each lane. (c) Growth curves of CD133positive and -negative subpopulation in SYO-1 treated by AKT inhibitor or DMSO, respectively. Data represent the number of viable cells and are means \pm s.d. of values from three independent experiments. ${ }^{* * *} P<0.005$ (Student's $t$-test) versus the samples except for CD133-negative subpopulation treated by DMSO. 


\section{Figure 1}

(a)
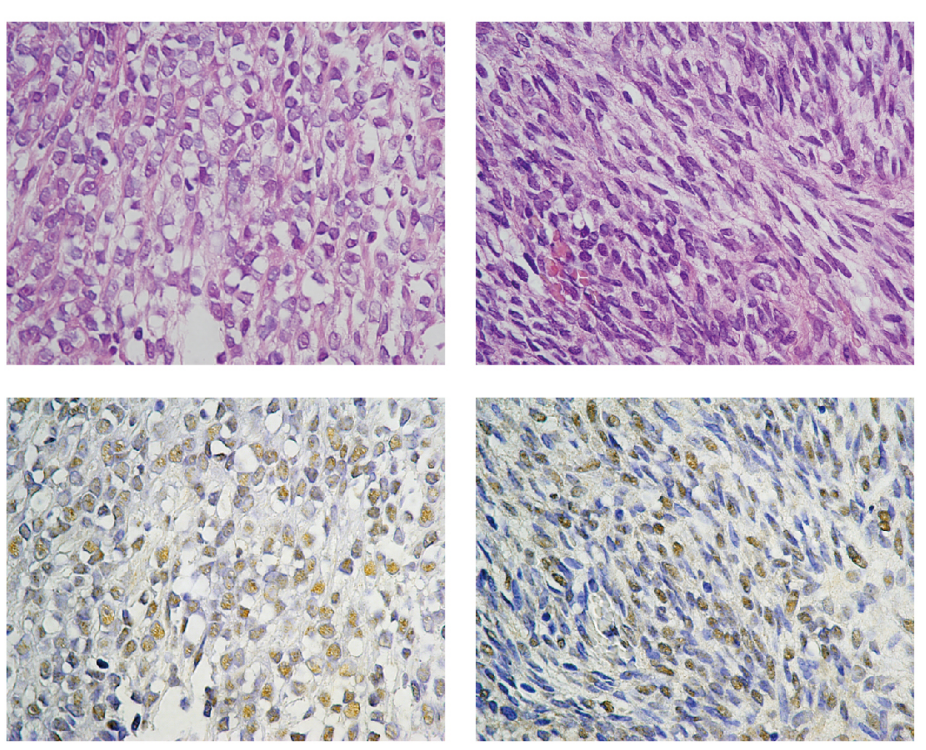

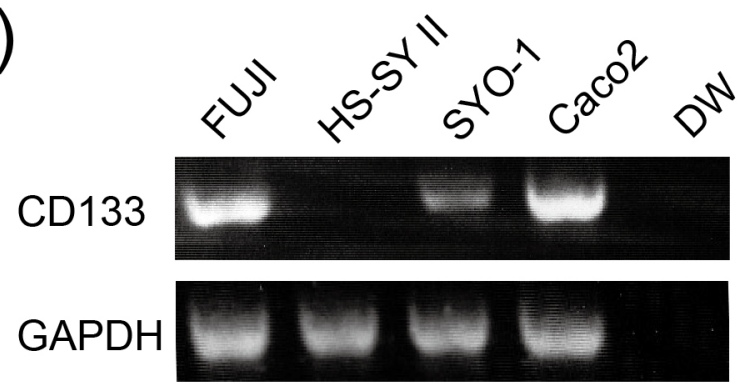

(c)

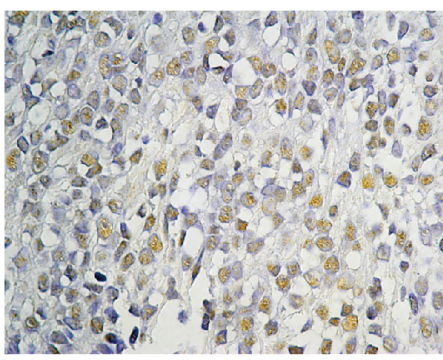

(b)

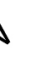

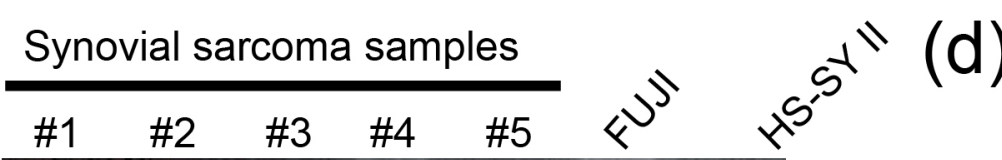

CD133

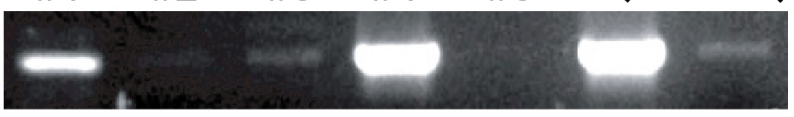

SS18-SSX1

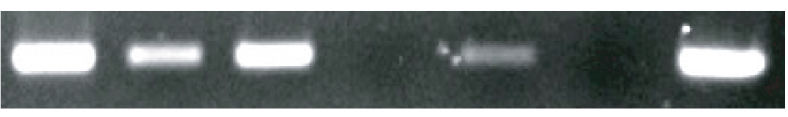

SS18SSX2

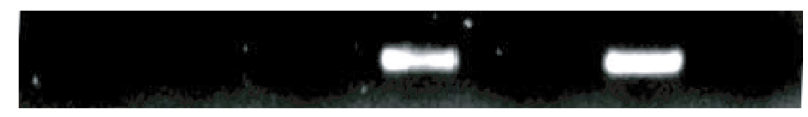

(d)

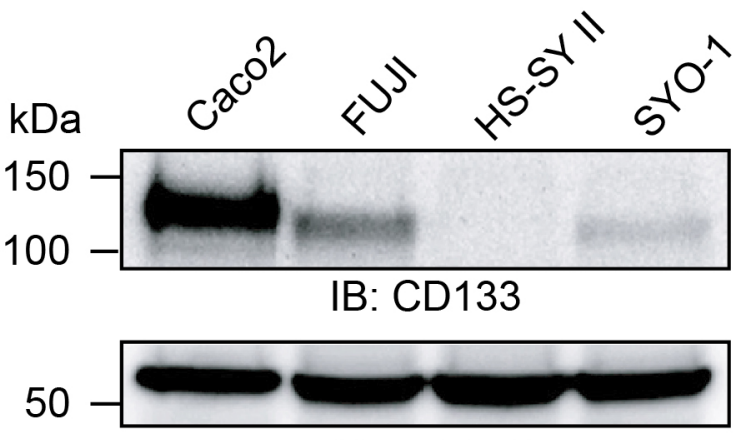

IB: $\alpha$ Tubulin

(e)
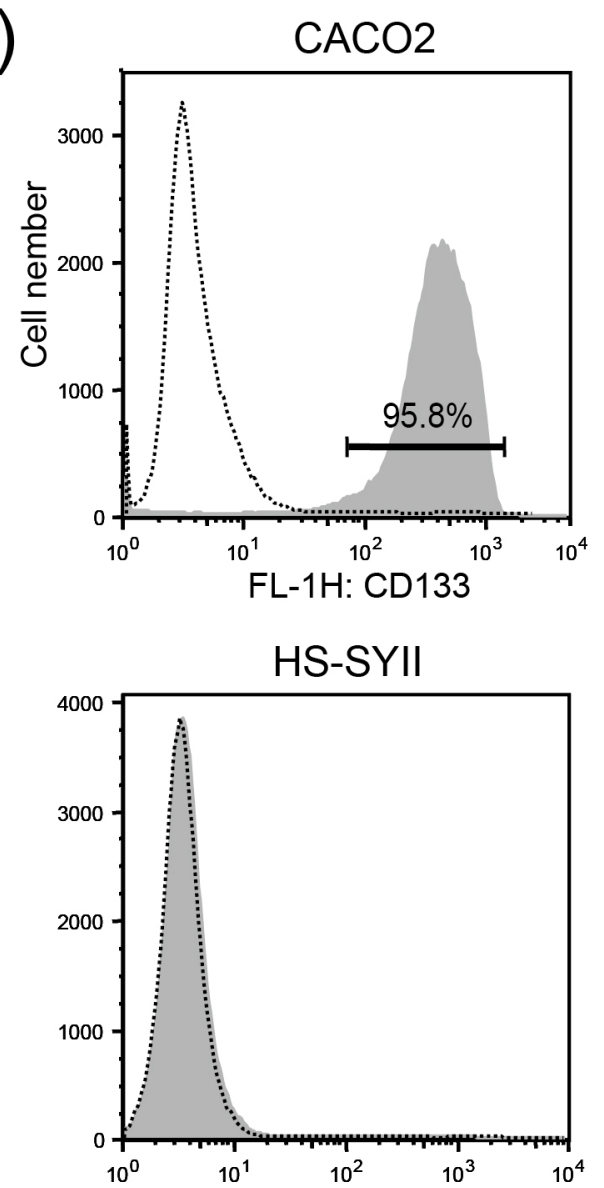

FUJI
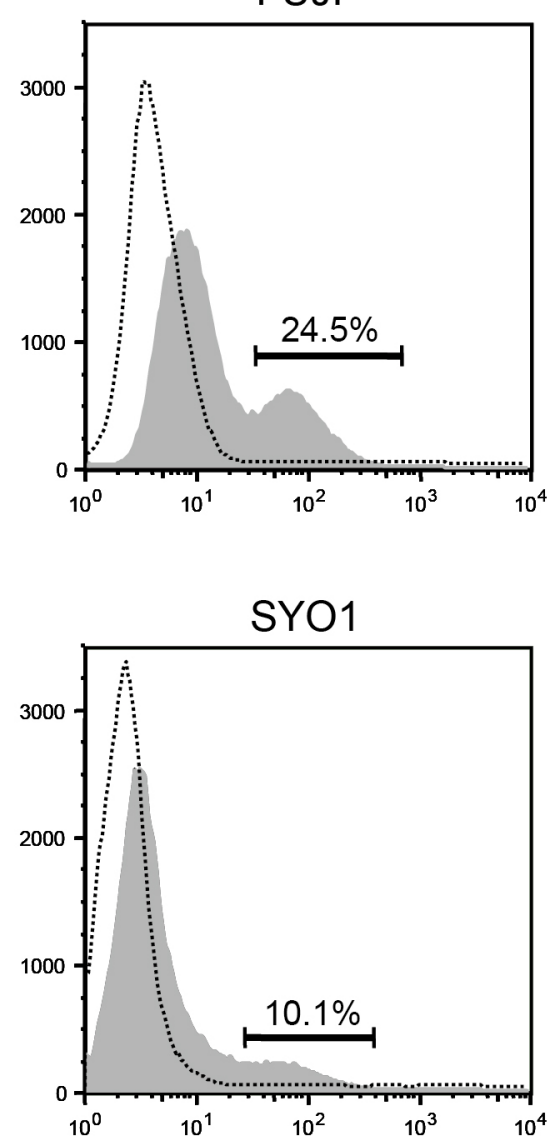
(a) SYO-1
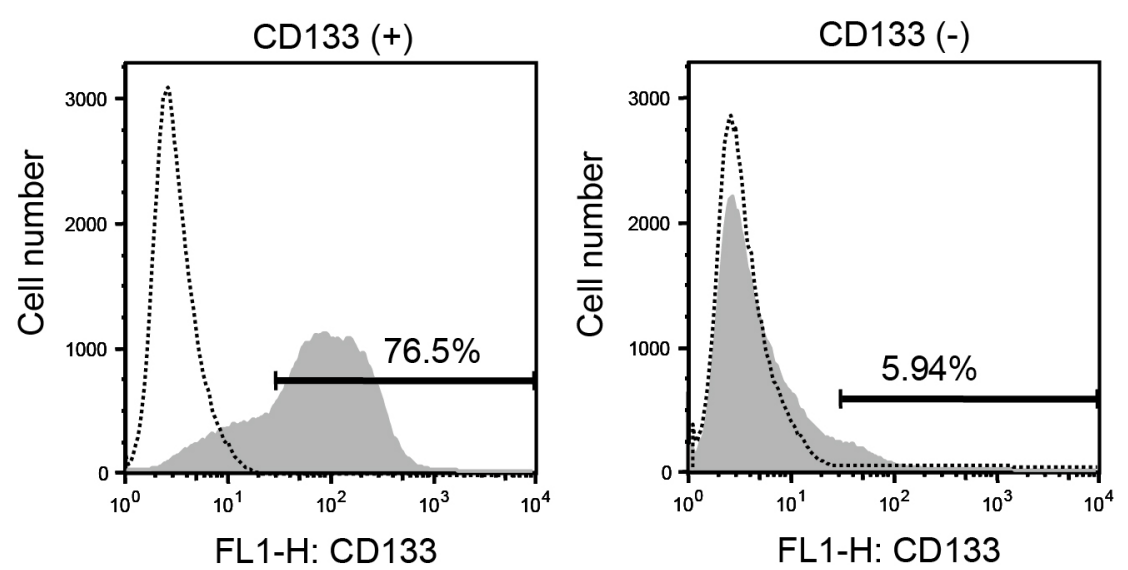

FUJI
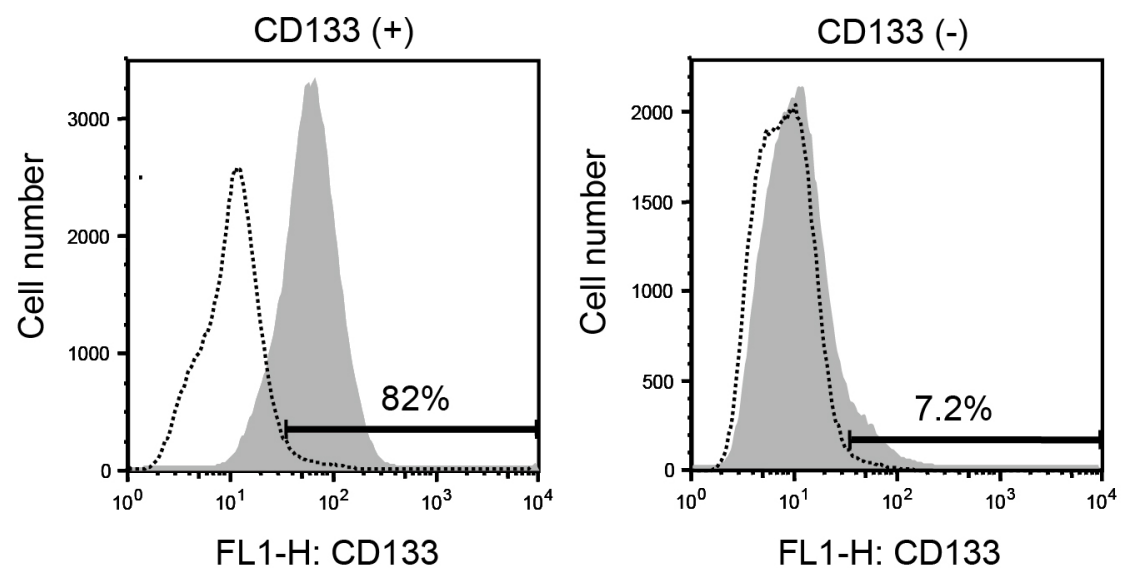

(b)

FUJI SYO-1 CD133 CD133

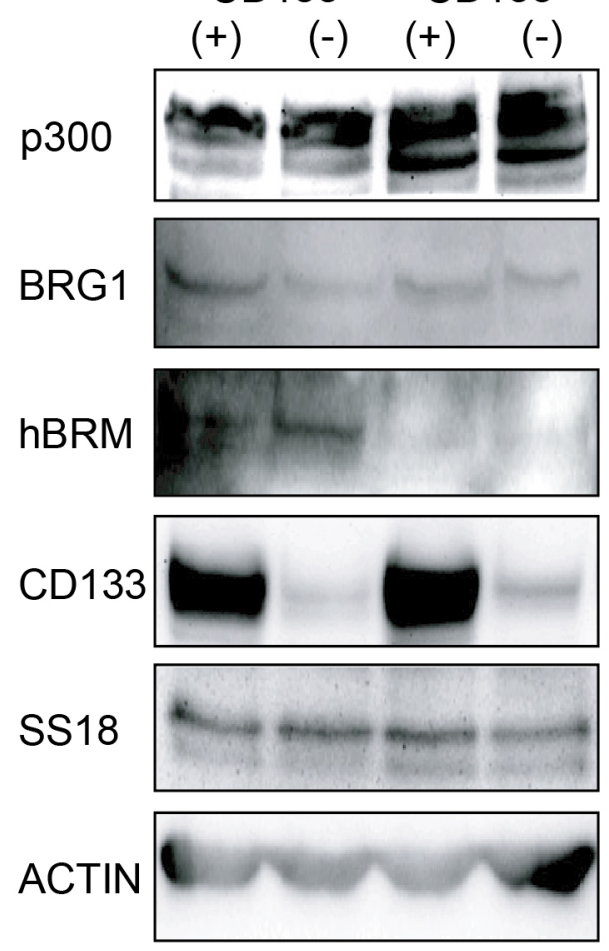

(c)

FUJI SYO-1

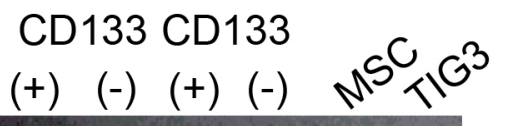

CD73 CD90 CD105 SS18 SS18-SSX2 CD133 GAPDH

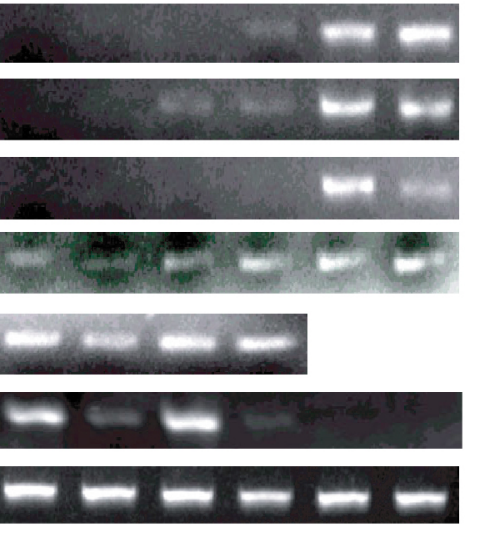


Figure 3

(a)

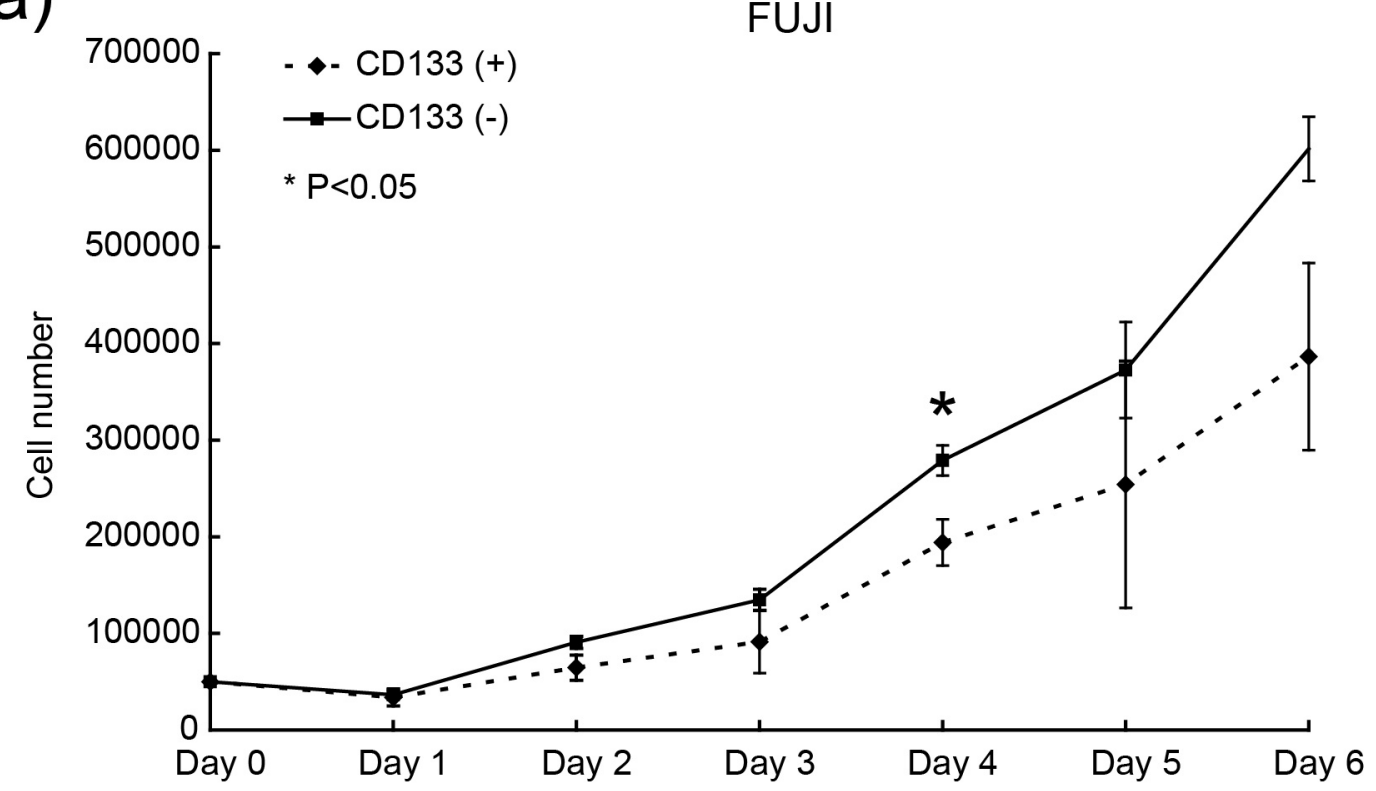

(b)

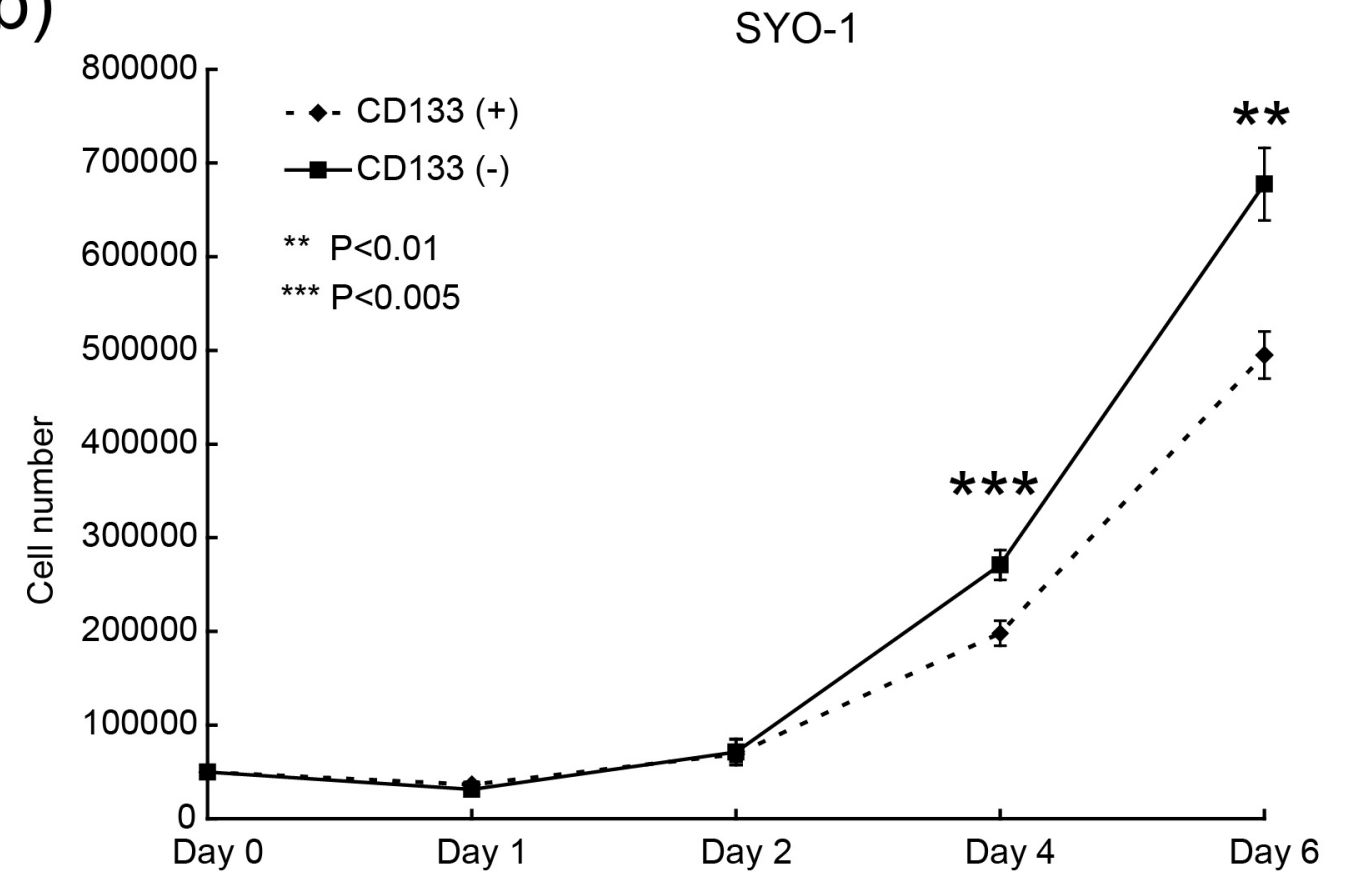

(c)
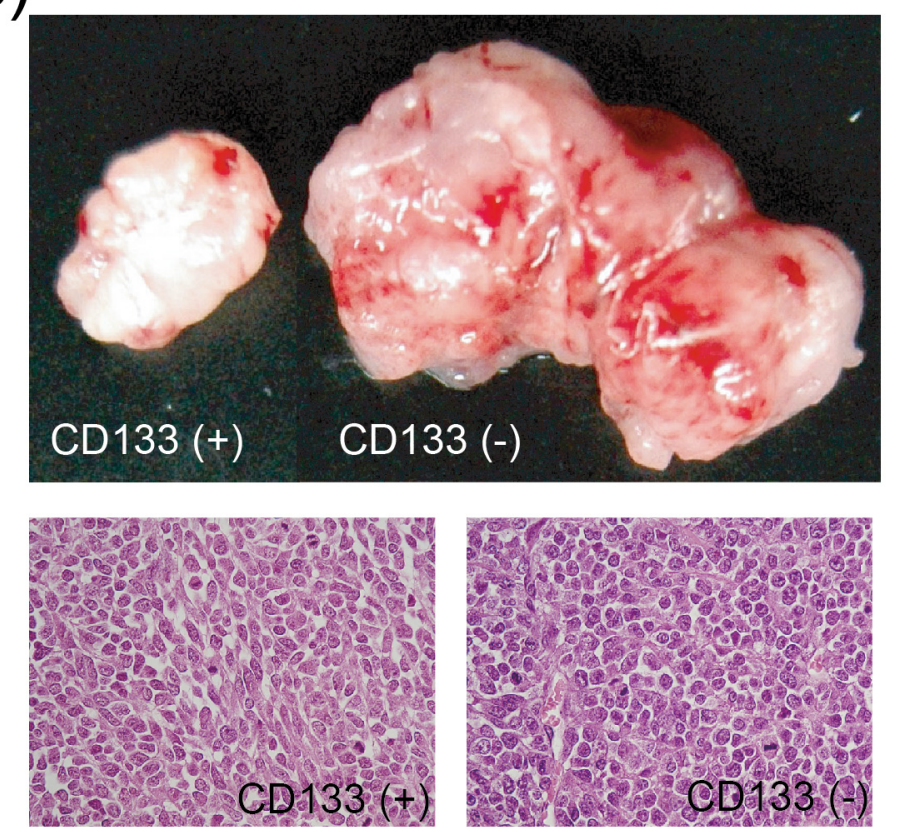

(d)

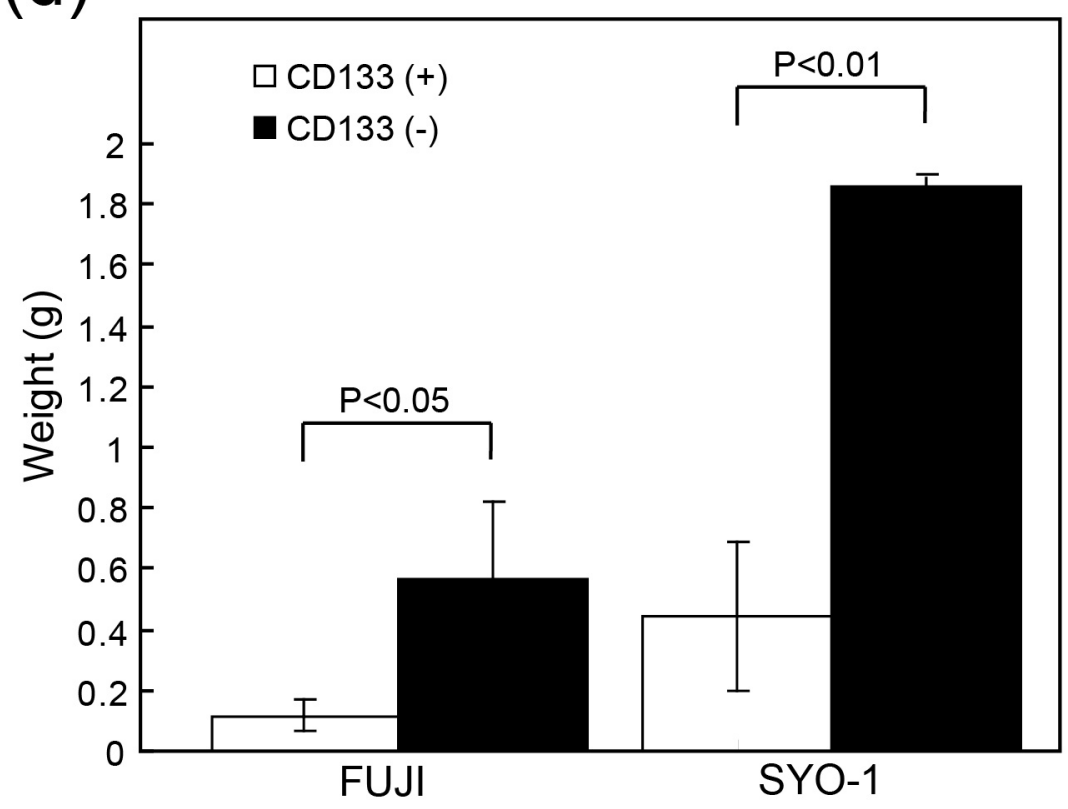




\section{Figure 4}

(a)

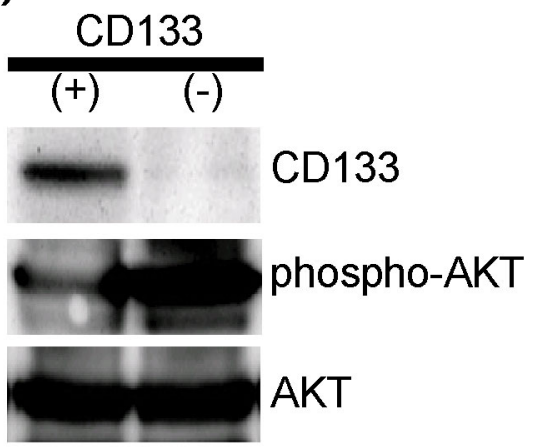

nise phospho-ERK

- phospho-JNK

(b)

$\frac{\text { CD133 }}{(+) \quad(-)}$

$\longrightarrow$ CDK1

CDK2

CDK4

CyclinD1

ACTIN
AKT inhibitor $(\mu \mathrm{M})$

$\begin{array}{llllll}\text { DMSO } & 0.1 & 0.5 & 1.0 & 2.0 & 5.0\end{array}$ phospho-AKT

phospho-cJUN

phospho-p38

(c)

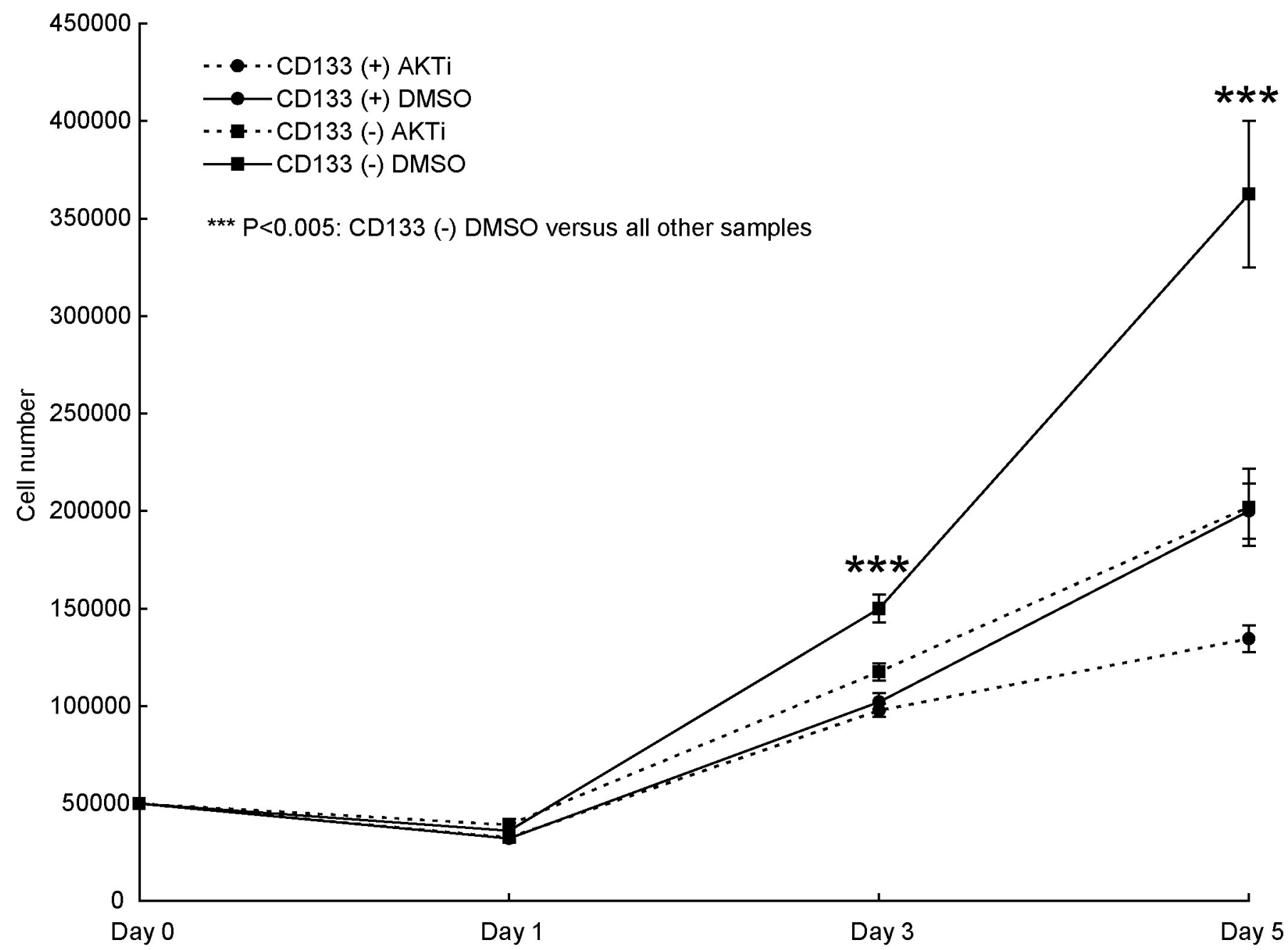

